\title{
Avaliação preliminar do comportamento da variação paleosecular durante o período de transição Paleozoico-Mesozoico
}

\author{
Felipe B.V. Freitas (ON/MCTI) ${ }^{1}$, Mariana B.S. Lebre (UFF) ${ }^{2}$, Daniel R. Franco (ON/MCTI) ${ }^{3}$ \\ ${ }^{1}$ Mestrando em Geofísica (bolsista CNPq), Observatório Nacional (ON/MCTI) \\ 2 Bolsista de Iniciação Científica CNPq/PIBIC \\ ${ }^{3}$ Coordenação de Geofísica, Observatório Nacional (ON/MCTI)
}

Copyright 2016, SBGf - Sociedade Brasileira de Geofísica

Este texto foi preparado para a apresentação no VII Simpósio Brasileiro de Geofísica, Ouro Preto, 25 a 27 de outubro de 2016. Seu conteúdo foi revisado pelo Comitê Técnico do VII SimBGf, mas não necessariamente representa a opinião da SBGf ou de seus associados. É proibida a reprodução total ou parcial deste material para propósitos comerciais sem prévia autorização da SBGf.

\section{Resumo}

In order to evaluate the stability of paleosecular variation (PSV) throughout the Paleozoic-Mesozoic transition interval, which happened after one of the geologic periods of lowest geomagnetic reversal rate - the PermoCarboniferous Reversed Superchron -, we recently started to conduct an analysis of the angular dispersion parameter of pre-selected virtual geomagnetic poles (VGPs) related to the $\sim 262-245 \mathrm{Ma}$ Permo-Triassic Mixed Polarity Hiperzone.

Preliminary results pointed out for higher angular dispersion datasets as a function of paleolatitude $(\lambda)$, accordingly to the expected for higher geomagnetic reversal rates, as discussed for the $~ 195-110 \mathrm{Ma}$ Jurassic-Cretaceous interval (McFadden et al., 1991), and predicted by some PSV $\times \lambda$ models (e.g., Model G; McFadden et al., 1998).

\section{Introdução}

O campo geomagnético (CG) apresenta variações em seu comportamento direcional e intensidade que vem sendo motivo de importantes debates ao longo das últimas décadas (Courtillot \& Le Mouel, 2007). Dentre os fenômenos que mais despertam interesse acerca do comportamento do geodínamo, estão as chamadas reversões de polaridade geomagnética, cujos mecanismos causais ainda são tema de importantes debates na literatura (Kruiver, 2000; Jacobs, 2001). Segundo alguns autores (e.g., Tarduno et al., 2002; Aubert et al., 2009), diferenças em taxas de reversão geomagnética observadas ao longo da GPTS (sigla em inglês para escala temporal de polaridade geomagnética) poderiam ser explicadas pela operação do geodínamo em regime de mais baixa energia do que, por exemplo, durante 'superchrons' - longos $\left(\sim 10^{7}\right.$ a) intervalos de polaridade simples do CG, e que poderiam ser causados quando processos geodinâmicos alteram o regime de fluxo térmico na região do núcleo externo e interface manto-núcleo, inibindo reversões (Merrill \& McFadden, 1994; Garcia et al., 2006).
Alguns estudos vêm discutindo que maiores dispersões de grupos de dados de PGVs vêm sendo discutidos para intervalos de alta frequência de reversão (Fig. 1), como no caso do Jurássico (McFadden et al., 1991); no caso de intervalos de baixa taxa de reversão, como no caso do SRPC ou do Superchron Normal do Cretáceo, têm sido verificados valores significativamente mais baixos (Biggin et al., 2008; Haldan et al., 2009; Franco et al., 2012). De acordo com Johnson e McFadden (2007), isto poderia ser explicado porque durante intervalos de alta taxa de reversões, componentes de ordem superior (e.g., quadrupolares, octupolares, etc.) responderiam como maior contribuição ao CG que a componente dipolar, com consequente reflexo na dispersão de polos geomagnéticos virtuais em função da (paleo)latitude conforme discutido por McFadden et al. (1998), que propuseram um modelo (Modelo G), baseado em expansão por harmônicos esféricos.

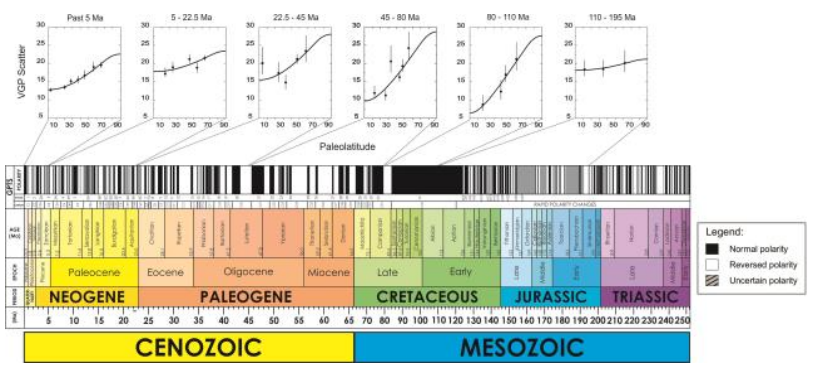

Figura 1 - Curvas de dispersão de PGVs relacionadas às paleolatitude para diferentes intervalos de tempo entre o Cenozóico e Mesozóico (com taxas de reversão distintas;), e comparados à Escala Global de Polaridade Geomagnética (GPTS).

Assim, investigações desta natureza, que possam avaliar os padrões de variação paleo-secular na transição entre intervalos de baixas para altas taxas de reversão, poderiam fornecer importantes subsídios aos atuais debates acerca da variação da estabilidade do geodínamo ao longo do tempo geológico.

Neste ínterim, o objetivo deste projeto é o de se avaliar a evolução do comportamento da variação paleo-secular (VPS) ao longo da transição Paleozóico-Mesozóico desde a Reversão Illawarra, abrangendo o intervalo relacionado à hiperzona de polaridade mista do PermoTriássico, até o Triássico Inferior - compondo a chamada Hiperzona de Polaridade Mista do Permo-Triássico (HPMPT). Tal esforço visa contribuir para as discussões 
sobre os processos geodinâmicos que governam os processos de reversão geomagnética.

\section{Metodologia}

Com o intuito de se avaliar os padrões de dispersão angular de PGV's em função da paleolatitude, desenvolveu-se um algoritmo que utiliza os dados paleomagnéticos de inclinação e declinação da magnetização remanente característica (MRC) proveniente da magnetização adquirida pelas rochas e sedimentos amostrados, durante a fase de sua formação em sitio - e as coordenadas dos sítios de amostragem. A partir dos dados de entrada são calculados os parâmetros estatísticos: declinação e inclinação média, latitude e longitude do polo paleomagnético, paleolatitude, parâmetro de dispersão angular $\left(\mathrm{S}_{\mathrm{B}}\right)$, e outros parâmetros estatísticos de interesse.

Os dados de entrada utilizados para os cálculos acima provenientes da literatura e que atendam aos critérios de qualidade aceitos como confiáveis pela comunidade científica internacional - são pré-selecionados de maneira a garantir a melhor aproximação possível dos valores reais procurados. Tais critérios abrangem, entre outros fatores, a metodologia de obtenção dos dados paleomagnéticos, e a qualidade dos dados direcionais de MRC. Para maiores detalhes acerca da metodologia de seleção de dados paleomagnéticos e o software desenvolvido, ver Assunção (2014).

\section{Resultados Preliminares e Conclusões}

Até o momento, foram selecionados dezenove estudos paleomagnéticos, que forneceram dados de dispersão angular de PGVs, associados às suas respectivas incertezas e valores de paleolatitude, em cobertura interhemisférica para o intervalo compreendido pela HPMPT (Fig. 2). Para efeitos de comparação com períodos que exibiram taxas de reversão distintas, e já discutidos na literatura (McFadden et al., 1991), tais resultados foram sobrepostos aos obtidos por este trabalho até o presente momento.

Embora a quantidade de informações para proporcionar uma melhor inferência a respeito do comportamento da variação paleo-secular (VPS) durante a HPMPT, uma análise preliminar do parâmetro de dispersão $\left(S_{B}\right)$ dos PGVs em função da paleolatitude, sugere valores de dispersão mais baixos para este intervalo do que para os demais períodos discutidos por McFadden et al. (1991).

É possível sugerir ainda uma baixa dependência dos valores do $S_{B}$ com os valores de paleolatitudes, o que é observado de maneira similar à curva correspondente ao período Jurássico-Cretáceo (195-110 Ma) e na curva obtida para o Paleoceno (5,0-22,5 Ma). Em ambos os

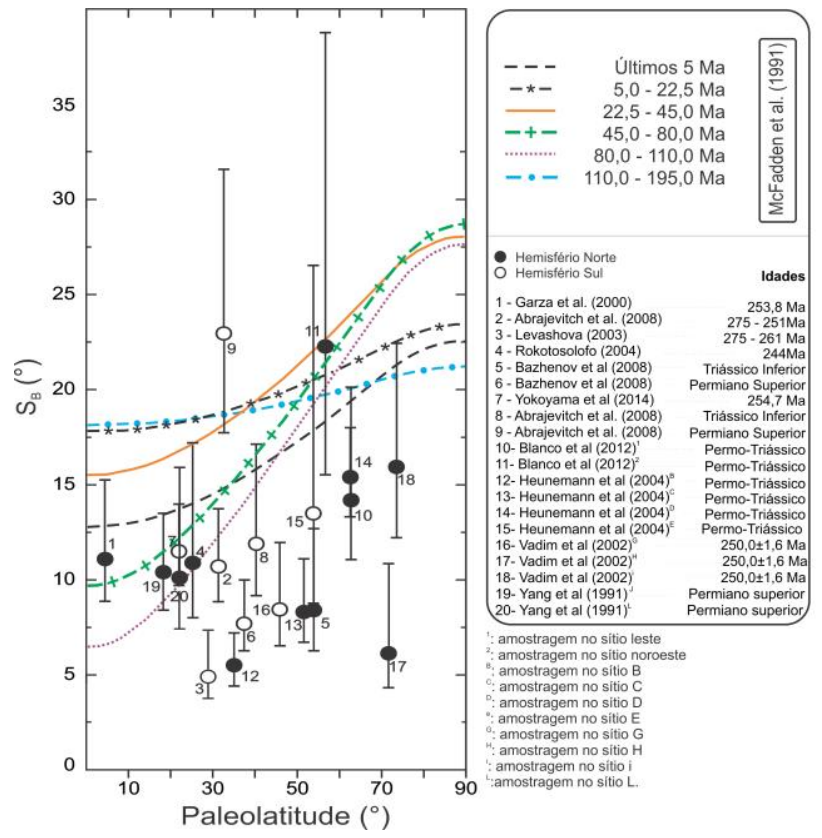

Figura 1: curvas ajustadas a dados de dispersão de PGVs para períodos de taxas de reversão geomagnética distintos (McFadden et al, 1991); dispersão de PGV's como função das paleolatitudes (este estudo).

casos, observa-se um resultado preliminar que corrobora a premissa adotada pelo Modelo $G$, isto é, durante períodos de grande instabilidade geomagnética menores contribuições da componente dipolar e maiores contribuições das componentes quadrupolares e octopolares - acompanhados de pouca dependência entre a dispersão de PGV's com as paleolatitudes. Neste momento, prosseguimos com o processamento de dados paleomagnéticos para refinar os resultados obtidos.

\section{Agradecimentos}

Felipe B.V. Freitas e Mariana B.S. Lebre agradecem, respectivamente, ao Conselho Nacional de Desenvolvimento Científico e Tecnológico (CNPq), pela concessão de suas bolsas de mestrado e de Iniciação Científica.

\section{Referências}

Assunção, K.S., 2014. "Estabilidade do Geodínamo: o problema dos "Superchrons". Monografia, Instituto de Física, Universidade Estadual do Rio de Janeiro, Rio de Janeiro, $67 \mathrm{p}$.

Aubert, J., Labrosse, S., Poitou, C., 2009. Modelling the palaeo-evolution of the geodynamo. Geophysical Journal International, 179 (3): 1414-1428.

Biggin, A. J., Hinsbergen D. J.J. van, Langereis C. G., Straathof G. B., Deenen M. H.L., 2008. Geomagnetic 
secular variation in the Cretaceous Normal Superchron and in the Jurassic. Physics of the Earth and Planetary Interiors, 169: 3-19.

Courtillot, V., Le Mouël, J.-L., 2007. The study of Earth's magnetism (1269-1950): a foundation by Peregrinus and subsequent development of geomagnetism and paleomagnetism. American Geophysical Union, Reviews of Geophysics, 45, RG3008, Paper \# 2006RG000198.

Driscoll P.E., Evans D. A. D., 2016. Frequency of Proterozoic geomagnetic superchrons. Earth and Planetary Science Letters, 437: 9-14.

Franco, D. R., Ernesto, M., Ponte-Neto, C. F., Hinnov, L. A., Berquó, T. S., Fabris, J. D., Rosière, C. A., 2012. Magnetostratigraphy and mid-palaeolatitude VGP dispersion during the Permo-Carboniferous Superchron: results from Paraná Basin (Southern Brazil) rhythmites. Geophysical Journal International, 191, n.3: 993-1014.

Garcia, A.S., Thomas, D.N., Liss, D., Shaw, J., 2006. Low geomagnetic field intensity during the Kiaman superchron: Thellier and microwave results from the Great Whin Sill intrusive complex. Geophysical Research Letters, vol. 33: L16308.

Haldan, M. M., Langereis, C. G., Biggin, A. J., Dekkers M. J., Evans M. E. A comparison of detailed equatorial red bed records of secular variation during the PermoCarboniferous Reversed Superchron. Geophysical Journal International, 177: 834-848.

Jacobs, J., 2001. The cause of superchrons. Astron. Geophys., 42, 6: 30-31.

Johnson, C.L., McFadden, P. L., 2007. Paleosecular Variation and the Time-Averaged Paleomagnetic Field. In: Kono, M., Treatise on Geophysics, vol. 5: 417-453, Elsevier, Amsterdam (2007).

Kruiver P.P., Dekkers M. J., Langereis C.G., 2000. Secular variation in Permian red beds from Dôme de Barrot, SE France. Earth and Planetary Science Letters Elsevier, 179: 205-217.

McFadden, P. L., Merrill, R. T., McElhinny, M. W., 1988. Dipole/quadrupole family modeling of paleosecular variation. Journal of Geophysical Research: Solid Earth, n. 93, B10: 11583-11588.

McFadden, P.L. Merrill, R.T. McElhinny, M.W. Lee. S., 1991 Reversals of the Earth's Magnetic Field and Temporal Variations of the Dynamo Families. Journal of Geophysical Research, vol 96, n. B3: 3923-3933.

Merrill, R.T., McFadden, P.L., 1994. Geomagnetic field stability: reversals events and excursions. Earth and Planetary Sciences, n. 121: 57-69.

Opdyke, N.D., 1995. Magnetostratigraphy of PermianCarboniferous time. Em: Geochronology Time Scales and
Global Stratigraphic Correlation, eds. Berggren, W.A., Kent,D.V. Aubry, M.-P. Hardenbol, J. Soc. Sed. Geol. Spec. Publ. (SEPM), Special Publications, 54: 41-47.

Tarduno, J.A., Cottrell, R.D., Smirnov, A.V., 2002. The Cretaceous Superchron geodynamo: Observations near the tangente cylinder. Department of Earth and Environmental Sciences, University of Rochester. Proceedings of the National Academy of Sciences, vol 99, n. 22: 14020 - 14025.

Van der Voo, R., 1990. The reliability of paleomagnetic data. Tectonophysics, vol. 184, n. 1: 1-9. 